\title{
Research on Nonlinear Characteristics of Hydro-pneumatic Suspension with Hydraulic Preload
}

\author{
Huichao Gao ${ }^{1,2}$, Xinyuan Chen ${ }^{1,2, *}$ \\ ${ }^{1}$ Key Laboratory of Metallurgical Equipment and Control Technology, Wuhan University of Science and Technology, Ministry of Education, \\ Wuhan, Hubei 430081, China \\ ${ }^{2}$ Hubei Key Laboratory of Mechanical Transmission and Manufacturing Engineering, Wuhan University of Science and Technology, \\ Wuhan, Hubei 430081, China
}

\begin{abstract}
The hydro-pneumatic suspension of special vehicles is mainly used to transmit vertical force and ease the impact and vibration caused by uneven road surface. As a new type of elastic element, the Hydropneumatic suspension generally uses inert gas or nitrogen as the elastic medium and hydraulic oil as the force transmission medium. Compared with ordinary leaf springs and coil springs, it has the advantages of small size and strong load-bearing capacity. This paper compares the Hydro-pneumatic suspension with hydraulic preload under different conditions and finds that under reasonable parameters, the hydro-pneumatic suspension can provide more stable performance.
\end{abstract}

\section{Introduction}

Suspension system is an important part of the vehicle. Its function is to elastically connect the body and the wheels, alleviate and attenuate the shock and vibration caused by uneven roads, and ensure the smooth driving of the vehicle. Traditional vehicles usually adopt the structure model of spring with shock absorber, while the hydro-pneumatic suspension is a combination of elastic elements and damping elements.

Hydro-pneumatic suspension is a special kind of suspension device in vehicle suspension. Compared with traditional vehicle passive suspension, it has the characteristics of large load and mass, variable stiffness and variable damping, and is generally used in heavy vehicles[1-3] and military vehicles[4]. Figure 1 shows a schematic diagram of a heavy vehicle with a hydropneumatic suspension.



Fig 1. A heavy vehicle equipped with a hydro-pneumatic suspension

Hydro-pneumatic suspensions can be divided into active hydro-pneumatic suspensions and passive hydro- pneumatic suspensions. The main research objectives of active hydro-pneumatic suspensions are control methods and strategies[5-8]. For example, optimal control theory, PID control theory[9], fuzzy control theory[10], adaptive control theory[11], robust control theory[12], genetic algorithm control theory, neural network control theory[13] and compound control theory. The research of passive hydro-pneumatic suspension is mainly aimed at the specific structure of the hydro-pneumatic suspension, the connection mode between various parts, and the changes in stiffness and damping under different conditions[14-17]. For example, Pevsner[18] first conducted theoretical research on interconnected hydro-pneumatic suspension system in 1957, and pointed out that interconnection has its suspension system, which can theoretically maintain good working characteristics by changing the connection method. Hou[19] studied the stiffness characteristics of the hydro-pneumatic suspension, and the results showed that it can improve the rolling resistance of the vehicle.

The previous research was carried out on the hydropneumatic suspension from the perspective of simulation, and most of the research objects were simple hydropneumatic suspensions, and did not discuss too much about hydro-pneumatic suspensions with hydraulic preload. In this paper, we focus on the stiffness characteristics and analyze its characteristics under different conditions.

\footnotetext{
*Corresponding author: cxy117@outlook.com
} 


\section{Suspension model establishing}

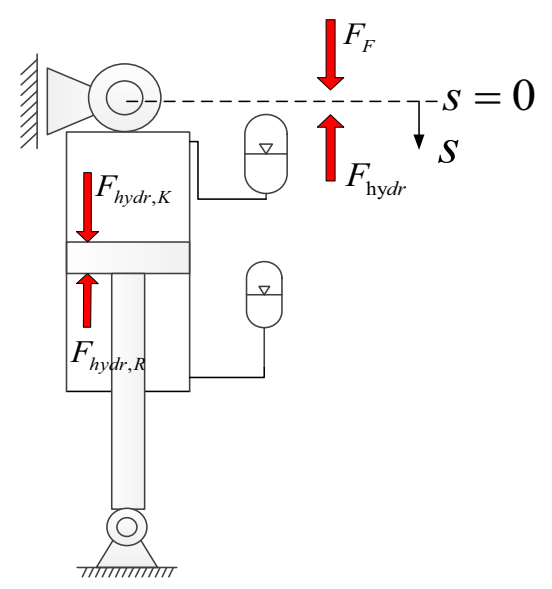

Fig 2. Schematic illustration of a hydro-pneumatic suspension with hydraulic preload

As shown in fig 2, the balance of forces on the rod is:

$$
F_{F(s)}=F_{h y d r, K(s)}-F_{h y d r, R(s)}
$$

The force calculation of the piston-side chamber $F_{\text {hydr,K(s) }}$ and rod-side chamber $F_{\text {hydr,R(s) }}$ as respectively as equation (2) and (3),

$$
\begin{gathered}
F_{h y d r, K(s)}=\left(F_{F 1}+F_{V}\right) \frac{\left(\frac{p_{0, K} V_{0, K}}{F_{F 1}+F_{V}}\right)^{n}}{\left(\frac{p_{0, K} V_{0, K}}{F_{F 1}+F_{V}}-s\right)^{n}} \\
F_{h y d r, R(s)}=F_{V} \frac{\left(\frac{p_{0, R} V_{0, R}}{F_{V}}\right)^{n}}{\left(\frac{p_{0, R} V_{0, R}}{F_{V}}+s\right)^{n}}
\end{gathered}
$$

$F_{\mathrm{F} 1}$, static spring load in design position $\mathrm{s}=0 ; F_{\mathrm{V}}$, preload force in design position, it results from the preload pressure $P_{\mathrm{V}}$ on the rod-side; $P_{0, \mathrm{~K}}, P_{0, \mathrm{R}}$, accumulators precharge pressure at room temperature $(293.15 \mathrm{~K})$; $V_{0, \mathrm{~K}}, V_{0, \mathrm{R}}$, internal accumulators volume; $s$, position of the piston, the displace $s$ in equations has different preceding algebraic signs, positive displacement means the piston-side is compressed, negative displacement means piston-side is expansion.

Combining all above equations brings us to

$$
F_{F(s)}=\left(F_{F 1}+p_{V} A_{R}\right) \frac{\left(\frac{p_{0, K} V_{0, K}}{F_{F 1}+p_{V} A_{R}}\right)^{n}}{\left(\frac{p_{0, K} V_{0, K}}{F_{F 1}+p_{V} A_{R}}-s\right)^{n}}-p_{V} A_{R} \frac{\left(\frac{p_{0, R} V_{0, R}}{p_{V} A_{R}}\right)^{n}}{\left(\frac{p_{0, R} V_{0, R}}{p_{V} A_{R}}+s\right)^{n}}
$$

The result for the suspension rate is $\mathrm{c}$ :

$$
\begin{gathered}
c=\frac{d F_{F(s)}}{d s}=n \cdot\left(F_{F 1}+p_{V} A_{R}\right) \cdot \frac{\left(\frac{p_{0, K} V_{0, K}}{F_{F 1}+p_{V} A_{R}}\right)^{n}}{\left(\frac{p_{0, K} V_{0, K}}{F_{F 1}+p_{V} A_{R}}-s\right)^{n+1}}+n \cdot\left(p_{V} A_{R}\right) \cdot \frac{\left(\frac{p_{0, R} V_{0, R}}{p_{V} A_{R}}\right)^{n}}{\left(\frac{p_{0, R} V_{0, R}}{p_{V} A_{R}}+s\right)^{n+1}}(5) \\
c_{S=0}=\frac{n\left(F_{F 1}+p_{V} A_{R}\right)^{2}}{p_{0, K} V_{0, K}}+\frac{n p_{V}^{2} A_{R}^{2}}{p_{0, R} V_{0, R}}
\end{gathered}
$$

System stiffness is equal to the sum of stiffness on both sides. The natural frequency can be calculated as:

$$
f=\frac{1}{2 \pi} \sqrt{\frac{\left(\frac{n\left(F_{F 1}+p_{V} A_{R}\right)^{2}}{p_{0, K} V_{0, K}}+\frac{n p_{V}^{2} A_{R}^{2}}{p_{o, R} V_{0, R}}\right) g}{F_{F 1}}}
$$

$n$, polytropic exponent.

It can be seen from the equations, $P_{0, \mathrm{~K}}, V_{0, \mathrm{~K}}, P_{0, \mathrm{R}}, V_{0, \mathrm{R}}$, $A_{R}$ and $P_{V}$ are available to tune the suspension to the desired properties. In the equations, these parameters always appear in pairs. $A_{R}$ and $P_{V}$ represent the hydraulic preload force, $P_{0, \mathrm{R}}$ and $V_{0, \mathrm{R}}$ represent the gas mass enclosed in the rod-side accumulator. These two sets of parameters represent the spring rate of the rod-side hydraulic system.

In this paper, total stroke of hydro-pneumatic suspension is $250 \mathrm{~mm}$. The center point of the total stroke is the static equilibrium point. The bearing mass of a single hydro-pneumatic suspension is about $61250 \mathrm{~N}$. the natural frequency of the hydro-pneumatic suspension is adjusted to $1.6 \mathrm{~Hz}$.

\section{Results and discuss}

Fig3, fig4 and fig5 shows the various characteristic curves of hydro-pneumatic suspension with hydraulic preload. All the curves are divided into two groups. The variable of one group is the preload force $(10 \mathrm{KN}, 15 \mathrm{KN}, 20 \mathrm{KN}$, $25 \mathrm{KN}$ ), and the variable of the other group is the rate of the rod chamber side $(20 \mathrm{~N} / \mathrm{mm}, 40 \mathrm{~N} / \mathrm{mm}, 60 \mathrm{~N} / \mathrm{mm}$, $80 \mathrm{~N} / \mathrm{mm})$.

Fig3 show the force-displacement curves of the hydropneumatic suspension under different conditions. It can be seen from the figure that the spring force and displacement present a nonlinear relationship, In the figure, the several curves in the shaded area almost overlap with no obvious difference. When the displacement continues to increase, the trend of the curve changes.

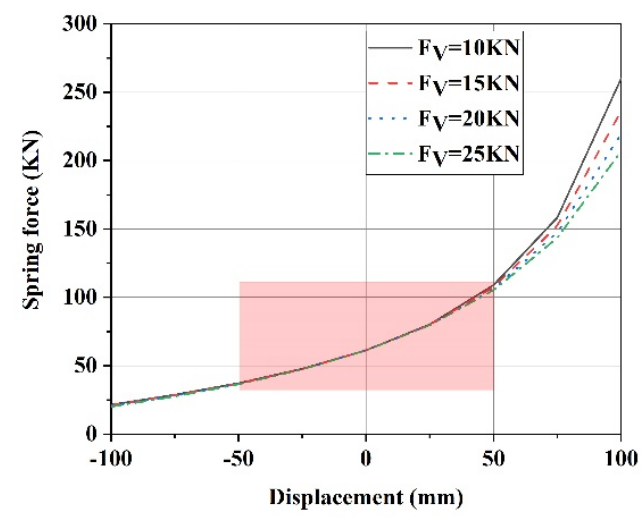

(a) 


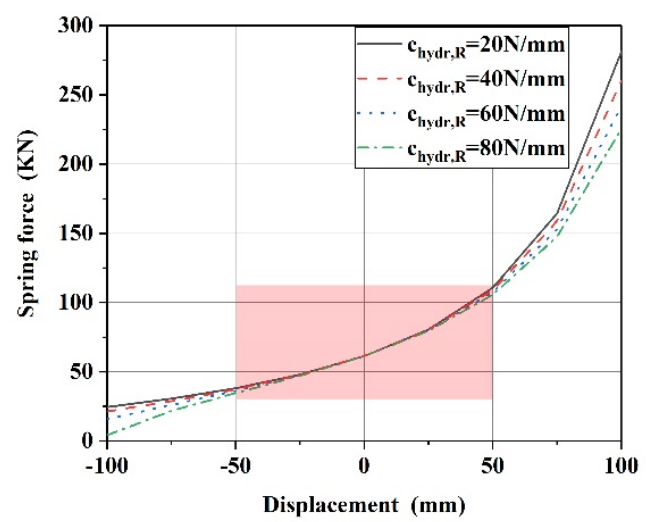

(b)

Fig 3. Force-displacement curves for the hydro-pneumatic spring with hydraulic preload. (a)Variable $F_{\mathrm{V}}\left(c_{\text {hydr, }}=40\right.$ $\mathrm{N} / \mathrm{mm})$. (b) Variable $c\left(F_{\mathrm{V}}=10 \mathrm{KN}\right)$.



(a)



(b)

Fig 4. Spring rate-static spring load curves for the hydropneumatic spring with hydraulic preload. (a)Variable $F_{\mathrm{V}}$ $\left(c_{\text {hydr, } \mathrm{R}}=40 \mathrm{~N} / \mathrm{mm}\right)$. (b) Variable $c\left(F_{\mathrm{V}}=10 \mathrm{KN}\right)$.

Fig 4 show the spring rate-static spring load curves of the hydro-pneumatic suspension with hydraulic preload under different conditions. As shown in the fig 3 and fig 4 , for hydro-pneumatic suspension with hydraulic preload, increasing the spring rate of the rod-side and increasing the preload have similar effects. When the displacement of the hydro-pneumatic suspension with hydraulic preload is small, the spring force is small and the rate of the suspension is also small, showing a better damping effect. As the weight carried on the suspension increases, the spring rate of the suspension also increases nonlinearly. At the same time, as the load increases, the rate growth of the hydro-pneumatic suspension under different preloads is also different. The static design point is the dividing point, and the curves on both sides have different behaviors.

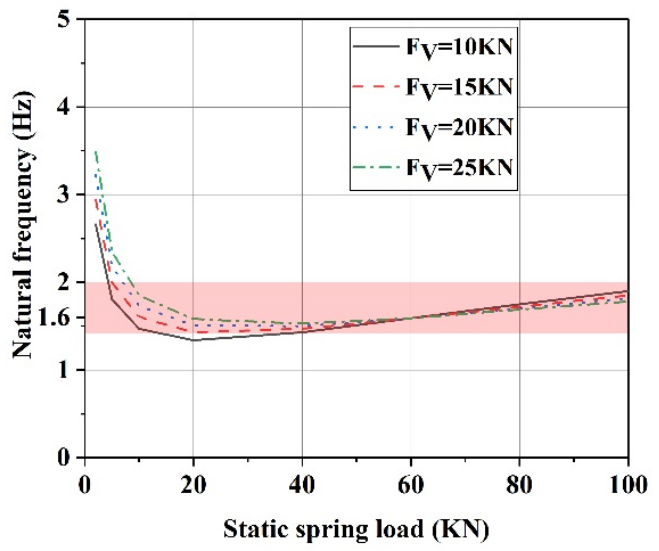

(a)

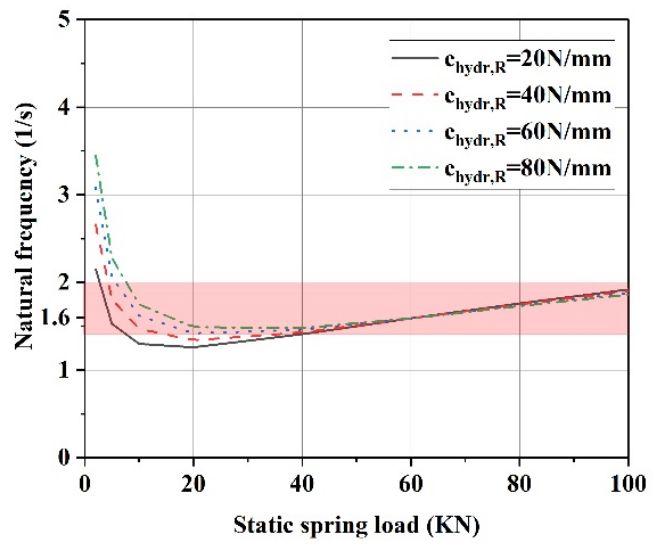

(b)

Fig 5. Natural frequency-static spring load curves for the hydro-pneumatic spring with hydraulic preload. (a)Variable $F_{\mathrm{V}}\left(c_{\text {hydr, } \mathrm{R}}=40 \mathrm{~N} / \mathrm{mm}\right)$. (b) Variable $c\left(F_{\mathrm{V}}=10 \mathrm{KN}\right)$.

In fig 5 , the natural frequency is maintained at an ideal level within a large load range, and even when the load is close to zero, the natural frequency will increase. The range of natural frequency is always maintained at 1.6-2 $\mathrm{Hz}$. With the increase of load mass, the natural frequency shows a trend of first decreasing and then increasing, but the natural frequency of the region from no load to full load performs better. From fig 5(a) and 5(b), it can be seen that regardless of whether the variable factor is the preload force $F_{\mathrm{V}}$ or the rate of the rod-side $C_{\mathrm{hydr}, \mathrm{R}}$, the trends of the curves in the two figures are similar. On the one hand, these are all due to the action of the preload force. Even when the load is zero, there is still preload force acting on the piston-side, hydro-pneumatic spring, and there is still available stiffness. On the other hand, it is a hydropneumatic suspension with hydraulic preload. The stiffness of the rod-side is still effective when the load is zero.

Increasing the preload by increasing the pressure of the rod-side will weaken the characteristic curve of the system 
without preload. The preload pressure $P_{\mathrm{V}}=0 \mathrm{MPa}$ would represents a system without preload force. Obviously as the preload force increases, the slope of the rate-load curve will decrease. Similarly, increasing the rate of the rod-side will reduce the rate of the whole system.

As shown in Figure 5(a), considering that the increase of the preload force will shift the minimum value of the natural frequency curve to a higher load position. Under high preload, the hydro-pneumatic suspension with hydraulic preload will significantly reduce the spring force of the entire system at the end of the return stroke. In Figure 4(b), we can see the influence of the rate of the rodside chamber on the whole rate of the hydro-pneumatic suspension. When the spring rate of the rod-side chamber is large, it means that when the stroke is close to the end of the rebound stroke, the gas volume in the rod-side accumulator is larger, and it is not easy to be compressed, which will make the spring force of the rod-side larger. By selecting appropriate parameters, the rate of the hydropneumatic suspension with hydraulic preload at the design position (center of stroke) can be very low, so that the suspension will be very soft in most of the working range, At the end, the spring force and the rate becomes larger, which can prevent the suspension from bottoming out.

\section{Conclusions}

The hydro-pneumatic suspension has a gradually increasing non-linear characteristic, that is, the rigidity of the suspension is small when the wheel travel is in a small range. When the vehicle is running on a better road, it can have better ride comfort; when the vehicle is running on a small undulating hard road, it can better alleviate the impact and vibration of the road. When driving on large undulating roads, as the wheel stroke increases, the suspension stiffness increases, which can effectively absorb external excitation energy, reduce the possibility of suspension breakdown, and improve comfort.

It can be seen from the above results that the hydropneumatic suspension can show better nonlinear characteristics no matter what the load condition is, and it has a wider applicability than ordinary mechanical springs. It can always keep the natural frequency of the suspension system near the design value. The research content of this article on hydro-pneumatic suspension can provide designers with certain reference value.

\section{Acknowledgments}

The project was supported in part by the National Natural Science Foundation of China under grants 51975425.

\section{References}

1. Kavhekar Gopiraj Parbhakar, Kulkarni S.N. IJRPET 3, 5 (2017)

2. Danish Ali, Samuel Frimpong. INT J IND ERGONOM $67,283-295(2018)$.

3. Kyuhyun Sim, Hwayoung Lee, Ji Won Yoon, Chanho Choi, Sung-Ho Hwang. Journal of Terramechanics
69(2017)

4. Massimiliano Gobbi, Giuseppe Marinaro, Gianpiero Mastinu, Giorgio Previati. ASME(2018)

5. Jifu G, Dongdong $\mathrm{H}$, Xinxia J. Journal of vibroengineering, 18, 1(2016)

6. Chen Zhou, Xinhui Liu, Feixiang Xu and Wei Chen. Appl. Sci. 10,1852(2020)

7. Tommaso Colombo, Giulio Panzani, Jorge de-J. Lozoya Santos and Sergio M. Savaresi. CCTA(2017)

8. M. D. Emami, S.A.Mostafavi, P. Asadollahzadeh. Mechanika, 17, 3(2011)

9. Heidari, M., Homaei, H., J. Eng. 2013, 1-9(2013).

10. Hathaway, R.J., Bezdek, J.C., 2001. IEEE Trans. Syst. 31, 735-744(2001).

11. J. Guan, L. Gu, and C. Hou, Acta Armamentarii, 25, 389-393(2004)

12. C. Crivellaro, In Proceedings of the SAE World Congress \&Exposition, vol. 2009.

13. Guarneri, P., Rocca, G., Gobbi, M., IEEE Trans. 19, 1549-1563(2008)

14. Xuefei Li, WeiDong Lv, Wei Zhang, Huanyu Zhao. Journal of vibroengineering. 19, 7(2017)

15. Yuming Yin, Subhash Rakheja, Jue Yang, Paul-Emile Boileau. Mechanical Systems and Signal Processing, 106, 319-333(2018)

16. Lukasz Konieczny. J VIBROENG, 17, 74-81(2015).

17. Ali, D., Frimpong, S., J. Powder Metall. Min. 6, 19(2017).

18. Pevsner J.m.,Automobile Engineer, Jan,10-16(1957)

19. N. Zhang, W. A. Smith, and J, Jeyakumaran, Vehicle Syst.Dyn.,48, 17-40(2010) 